\title{
Healing Effect of Platelet-rich Plasma on Decellularized Tracheal Allotransplantation in Rabbits
}

\author{
SEOK JIN JANG, MIN-HO PARK, TAE-KI LEE and SEOK HWA CHOI \\ Department of Veterinary Surgery, College of Veterinary Medicine, \\ Chungbuk National University, Cheongju, Republic of Korea
}

\begin{abstract}
Background/Aim: The purpose of this study was to explore the effect of platelet-rich plasma (PRP) on enhancing healing of trachea allotransplantation and confirm the effect via parallel histological and tracheoscopic examinations in seven adult New Zealand White rabbits. Materials and Methods: Harvested trachea was inserted into recipients with end-to-end anastomosis by a simple interrupted suture. PRP-treated rabbits were treated with $0.5 \mathrm{ml}$ of PRP at the trachea grafts, while control rabbit allografts were treated with $0.5 \mathrm{ml}$ of saline. Results: Tracheoscopy of tracheal allografts treated with PRP revealed that the trachea was well healed with no stenosis. The healing effect in the PRP-treated rabbits increased tracheal activity and produced faster trachea regeneration compared to that in control rabbits. There was a good correlation between the subjective symptom of noisy breathing and the objective grading of tracheal stenosis. The tracheal allografts with suture materials appeared slightly pale and looked more like mucosa erosion than normal mucosa at four weeks post-surgery. Contact of trachea-totransplanted grafts in PRP-treated rabbits was intimate with the surface of the transplanted region and showed highdensity epithelialization. After 8 weeks, blood vessels were observed in the transplanted graft in PRP-treated rabbits. Normal epithelium was present in grafts at 8 weeks after allotransplantation. No $\mathrm{CD} 2 \mathrm{O}^{+}$cells were detected in grafts but a few $\mathrm{CD}^{+}$cells were observed under the epithelium. Conclusion: The results of this study show that it is possible
\end{abstract}

This article is freely accessible online.

Correspondence to: Professor Seok Hwa Choi, DVM, Ph.D., Department of Veterinary Surgery, College of Veterinary Medicine, Chungbuk National University, Cheongju 28644, Republic of Korea. Tel: +82 432613144, Fax: +82 432613224, e-mail: shchoi@cbu.ac.kr

Key Words: Decellularized trachea, allotransplantation, platelet-rich plasma, rabbit. to perform tracheal reconstruction in rabbits treated with PRP after tracheal transplantation.

Tracheal reconstruction after segmental resection of stenotic lesions or a malignant tumor is one of the most difficult surgical procedures to perform. Tracheal lesions are often treated with tracheal allografts or via regeneration due to stenosis of the respiratory tract (1). Stenosis of the trachea causes significant morbidity and mortality. Short segmented tracheal lesions $(<5 \mathrm{~cm})$ can be excised with end-to-end anastomosis in human medicine. However, it is challenging to repair a long-segmental tracheal lesion $(>5 \mathrm{~cm})$ via immediate repair of the long-segmental defects using trachea allografts or tracheal regeneration (2). Unfortunately, the resectable length of the diseased trachea is usually restricted to approximately $30 \%$ of the total length in children and to around $6 \mathrm{~cm}$ length in adults. A tracheal stenosis longer than $50 \%$ of the total tracheal length is generally considered unresectable. In such a case, replacement of the trachea is necessary. There are several methods by which to replace a defective trachea, and the ideal material for tracheal replacement is autogenous tissue. It has been reported that direct tracheal transplantation is impossible due to the lack of a well-defined blood supply for vascular anastomosis during tracheal surgery (2). Thus, the isolated trachea is wrapped in a sufficiently vascularized soft-tissue flap to achieve intratracheal revascularization. After full revascularization and mucosal regeneration are achieved (within 2-4 months), trachea transplantation can be performed (2). The trachea is one of the few organs that are exceptionally difficult to transplant because of the technical difficulty associated with restoring a blood supply to the graft (3).

Platelet-rich plasma (PRP) is used in periodontal diseases, maxillofacial and orthopedic surgeries, and sports medicine (4). Recently, it has attracted attention in skin disease, wound healing, and hair regrowth studies (5). PRP or platelet concentrates are inexpensive products, easy to obtain, and they include important growth factors involved in tissue repair. An example of such growth factors are the alpha granules of activated platelets, which stimulate chemotaxis, 
fibroplasia and angiogenesis (6). In this study, the healing effect of PRP on decellularized tracheal allotransplantation in rabbits was investigated.

\section{Materials and Methods}

Preparation of PRP. Rabbits were divided into two groups ( $\mathrm{n}=7$ per group): PRP-treated rabbits were treated with $0.5 \mathrm{ml}$ of PRP at the trachea grafts, while control rabbit allografts were treated with $0.5 \mathrm{ml}$ of saline. Peripheral venous blood was obtained prior to the administration of anesthetics. Seven $5 \mathrm{ml}$ tubes containing EDTA as an anticoagulant were drawn from each recipient rabbit. The tubes were centrifuged at $268 \times g$ for $20 \mathrm{~min}$ at room temperature. The blood was separated into three parts: red blood cells (RBCs), PRP, and platelet-poor plasma. The portion corresponding to the platelet-poor plasma was ejected from each tube and the remaining content was centrifuged again at $448 \times g$ for $15 \mathrm{~min}$. The middle portion, corresponding to PRP, was pipetted from each tube and $10 \%$ calcium chloride was added to the preparation to activate the platelets.

Decellularized trachea preparation and surgical procedures. Fourteen 6-month-old male New Zealand White rabbits (Samtaco Lab., Osan, Korea), weighing approximately $3.1 \mathrm{~kg}$, were used for this study. Rabbits were placed in individual cages and fed water and standard diet ad libitum. The protocol for animal experiments was approved by the IACUC Laboratory Animal Research Center of Chungbuk National University (CA-17-21). Seven healthy rabbits were used as trachea tissue recipients and another seven rabbits were used as donors. Tissues and muscles on the harvested trachea were stripped and the trachea rinsed four times for $4 \mathrm{~h}$ each in phosphate-buffered saline containing $1 \%$ antibiotic and antimycotic solution. Harvested tissues were stored in Aqua milli-Q (Millipore ${ }^{\circledR}$, Sigma-Aldrich Corporation, St. Louis, MO, USA) for $48 \mathrm{~h}$ at $4^{\circ} \mathrm{C}$ and incubated for $4 \mathrm{~h}$ with $2,000 \mathrm{kU}$ of DNase-I in $4 \%$ sodium deoxycholate and $1 \mathrm{~mol} / \mathrm{l} \mathrm{NaCl}$ (Sigma-Aldrich Corporation). The presence of cellular elements was confirmed through histological examination.

The seven donor rabbits were placed in a supine position under general anesthesia and the 11th to 16th tracheal rings, $36.59 \mathrm{~mm}$ in length, were excised as a tube shape through a midline cervical incision (Figure 1). Recipient rabbits under general anesthesia were placed in a supine position and the harvested trachea was inserted via end-to-end anastomosis by using a simple interrupted suture of absorbable monofilament (5-0 PDS, Ethicon, UK). The PRP-treated rabbits had $0.5 \mathrm{ml}$ of PRP deposited into the trachea graft, while control rabbits had $0.5 \mathrm{ml}$ of saline introduced into the allografts. Rabbits were intravenously administered anti-thymocyte globulin (2.5 mg/kg, Thymoglobulin; Genzyme, Cambridge, MA, USA) and methylprednisolone sodium succinate $(1 \mathrm{mg} / \mathrm{kg}$, Primedrol inj $125 \mathrm{mg}$; Jeilpharm, Seoul, Korea) on the first and second days after surgery. Tacrolimus (4.5 mg/kg, Prograf; Astellas Pharma Korea, Seoul, Korea) was orally administered twice a day for 4 weeks.

Tracheoscopic evaluation. Tracheoscopic evaluation was performed in rabbits for 8 weeks after tracheal graft implantation. Tracheoscopy was undertaken with a 4-mm rigid endoscope (Karl Storz, Tuttlingen, Germany) and a video-endoscopy system consisting of a video tracheoscope (OTV-S70 Pro; Olympus Co Ltd, Tokyo, Japan) and a video processor coupled to a light source (CLV-S40 Pro; Olympus Co Ltd). The degree of tracheaI stenosis was analyzed by calculating the strictured portion as a percentage of the native tracheal diameter by using the image processing program 'ImageJ', a free, open-source image processing platform supported by the National Institutes of Health (Wayne Rasband, National Institute of Mental Health, Bethesda, MD, USA). Based on the results of these calculations, tracheal stenosis was classified into four subgroups: Grade I: $<25 \%$ obstruction; grade II: $25-50 \%$ obstruction; grade III: $50-75 \%$ obstruction; and grade IV: $>75 \%$ obstruction. The subjective symptom of noisy breathing and the objective grade of tracheal stenosis were compared 8 weeks after transplantation (7).

Histological examination. Grafts of rabbit trachea were used for histological examination. The samples were fixed in $4 \%$ paraformaldehyde in phosphate-buffered saline and embedded according to routine paraffin-embedding protocols. Paraffinembedded tissues were sectioned at $4 \mu \mathrm{m}$ using a microtome. The prepared sections were stained with hematoxylin and eosin (H\&E), and antibodies to T-cell co-receptor CD3 (rabbit polyclonal, DAKO, Glostrup, Denmark), and activated-glycosylated phosphoprotein CD20 expressed on the surface of all B-cells (mouse monoclonal; Thermo Scientific, Waltham, CA, USA). The sections were incubated with a primary antibody cocktail designed for each target. The sections were subsequently incubated with a secondary antibody cocktail of anti-rabbit/horseradish peroxidase (HRP+) antimouse/alkaline phosphatase (AP) polymers. For color development, the slides were incubated with blue chromogen (Thermo Scientific) for AP and 3,3'-diaminobenzidine chromogen (DAKO, Glostrup, Denmark) for HRP. The stained samples were qualitatively observed under an Axio Imager A1 microscope, and micrographs were obtained by using Axio-Vision software (Carl Zeiss AG, Oberkochen, Germany).

Statistical analysis. Statistical analyses were performed using SPSS statistical software package version 19.0.1.1. (IBM SPSS Statistics for Windows, Version 19.0; IBM Corp., Armonk, NY, USA). Data are presented as mean \pm standard deviation (SD) values. Normality and homogeneity of the data were confirmed before analysis of variance (ANOVA). Differences among the experimental groups were assessed by one-way ANOVA followed by Duncan's multiple range tests. Null hypotheses of no difference were rejected if $p$ values were less than 0.05 .

\section{Results}

Blood smears. Platelet counts for each rabbit yielded a mean platelet count of $382,000 / \mu 1$ (range $=299,000-441,000 / \mu 1)$. The mean platelet count of the PRP fraction was $1,157,000 / \mu \mathrm{l}$ (range $=1,039,000-1,452,000 / \mu \mathrm{l})$. These values confirmed the utility of the process and quantified the count as being $334 \%$ of the baseline platelet count.

Tracheoscopy observations in recipient rabbits. Tracheoscopy at 1 week after transplantation revealed that the interface between the natural trachea and the transplanted graft was covered with granulation tissue. At 2 weeks, the granulation tissue at the interface had partially regressed. However, after 4 weeks of implantation, the interface was covered with regenerated epithelium. Overall, 6/7 (86\%) of the control rabbit group showed grades I and II stenosis, but 5/7 (71\%) 

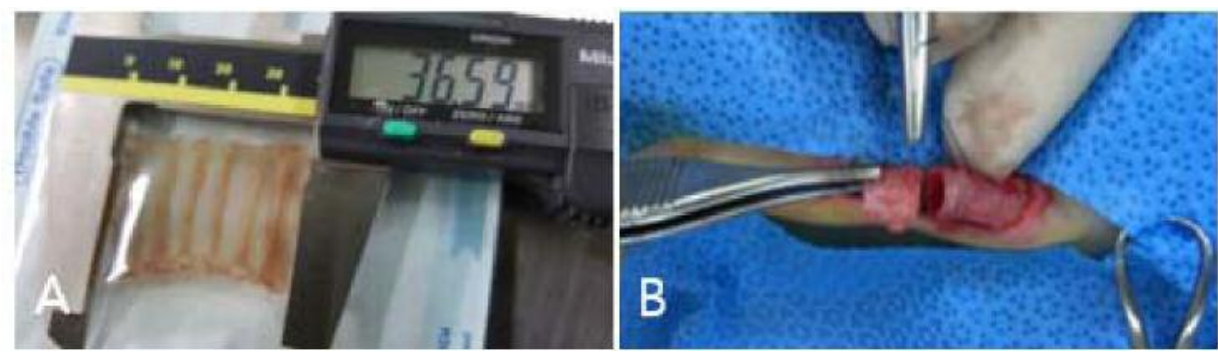

Figure 1. Image showing tracheal size (A) and intraoperative image of tracheal allotransplantation (B) in New Zealand White rabbits.
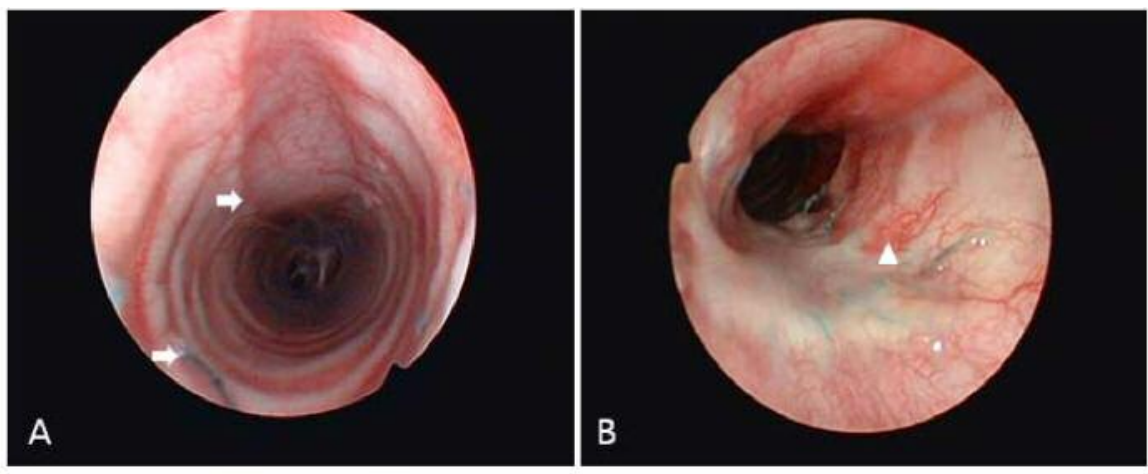

Figure 2. Tracheoscopic images of a tracheaI allotransplantation region (arrows) at 4 (A) and 8 weeks (B) after platelet-rich plasma (PRP) treatment in New Zealand White rabbits. At 8 weeks after PRP treatment, blood vessels (arrowhead) were observed at the transplanted graft.

of the PRP-treated rabbit group showed grade I stenosis (Table I). The comparison of the subjective symptom of noisy breathing and the objective grading of tracheal stenosis revealed a good correlation. All rabbits with noisy breathing had grade I or II tracheal stenosis. The tracheal graft site with suture materials appeared to be slightly pale and looked as though there was mucosal erosion present rather than normal mucosa at 4 weeks (Figure 2A). The surface of the transplanted allograft showed the presence of blood vessels at 8 weeks after surgery (Figure 2B).

Histological examination results. Based on H\&E staining results, normal epithelium was present in the grafts at 8 weeks after transplantation (Figure $3 \mathrm{~A}$ ). No $\mathrm{CD} 20^{+}$cells were observed in the grafts and few $\mathrm{CD}^{+}$cells were observed under the epithelium. Lymphocyte infiltration was not observed, neither in the epithelial layer nor underneath (Figure 3B).

\section{Discussion}

In this study, trachea allotransplantation was performed as an end-to-end anastomosis. End-to-end anastomosis repair is the gold standard surgical repair technique after tracheal
Table I. Endotracheal diameter $(\mathrm{mm})$ at transplanted grafts 8 weeks after tracheaI allotransplantation. Data are presented as mean $\pm S D$ $(n=7)$.

\begin{tabular}{lccc}
\hline Rabbits & Pre-operative & \multicolumn{2}{c}{ Post-operative } \\
\cline { 3 - 4 } & & $\begin{array}{c}\text { Inner vertical } \\
\text { diameter }\end{array}$ & $\begin{array}{c}\text { Inner transversal } \\
\text { diameter }\end{array}$ \\
\hline Control & $5.81 \pm 0.38$ & $4.17 \pm 0.82$ & $4.35 \pm 0.75$ \\
PRP-treated & $5.82 \pm 0.34$ & $4.84 \pm 0.91^{*}$ & $4.96 \pm 0.98 *$ \\
\hline
\end{tabular}

*Significantly different at $p<0.05$ from the control group.

segmental resection for benign or malignant diseases (8). TracheaI grafts were observed by tracheoscopy in order to monitor stenosis of the implanted grafts. Grade II stenosis was not as severe as expected in the PRP-treated rabbits. However, on tracheoscopy, healing effects in both control and PRP-treated rabbits were clearly observed. The tracheal epithelium provides a structural and functional barrier, prevents inhaled substances from entering the trachea, and 




Figure 3. Histological images of grafts at 8 weeks after tracheaI allotransplantation. A: The transplanted graft region was observed to be covered with fibrous tissue with re-epithelialization and fibroproliferation in rabbits (hematoxylin and eosin, $\times 10$ ). B: Presence of a few CD $3^{+}$cells in the epithelium on immunohistochemical staining $(\times 400)$.

has an important role in regulating the activity of innate immune systems (9). Therefore, the early regeneration of epithelium in transplanted tissue is the most important factor in tracheal reconstruction (10).

Based on H\&E staining results, normal epithelium was present in the graft at 8 weeks after transplantation. Lymphocyte infiltration was not observed in the epithelium or underneath it. The immunohistochemical observations at 8 weeks showed a normal tracheal epithelium with few infiltrations of $\mathrm{CD}^{+}$cells, which appeared to be normal resident T-cells in the mucosa. These findings were the result of epithelial regeneration in the transplanted grafts. In a previous study, epithelial regeneration in animal models showed that respiratory epithelium formed by dedifferentiating, spreading, migrating, and gradually redifferentiating to reconstitute on the shedding basement membrane after several weeks (11). In addition, $\mathrm{CD}^{+}$and $\mathrm{CD} 20^{+}$cells in the immunohistochemical examination of the present study showed that PRP-treated rabbits had greater new epithelialization than did control rabbits.

In this study, steroids and tacrolimus were mainly used as immunosuppressive agents for T-cell targets. If immunosuppressive agents for antibody control are incorporated, rabbit tracheal grafts would appear normal by tracheoscopy at 8 weeks after surgery. This phenomenon is similar to the healing mechanism associated with mucosal erosion in tracheal allografts without immunosuppression (12). We did not use specific immunosuppressants to control anti-rabbit antibodies. The reason for this is that low antigenicity of rabbit cartilage cells may not lead to cartilage rejection.
Of the cells in a typical blood sample, $93 \%$ are RBCs, $6 \%$ are platelets, and $1 \%$ are white blood cells. The principle behind the PRP process is to concentrate the platelets by centrifugation to reverse the RBC:platelet ratio and create a sample with $94 \%$ platelets and 5\% RBC. Platelets are most frequently associated with a hemostatic function. However, they also contain more than 800 active reservoirs acting on multiple targets, including secretory cells, stem cells, fibroblasts, osteoblasts, endothelial cells, and epithelial cells $(13,14)$. Granulation of these factors generally begins within $10 \mathrm{~min}$ of platelet activation. In addition to platelets and their secreted factors, other active components, especially fibrinogen and white blood cells, are present in PRP (13). PRP is an interesting product for cell-based therapy and tissue engineering. Some researchers have described various beneficial effects of PRP including proliferation of fat precursor cells, wound repair, cell differentiation, and angiogenesis (14). Moreover, PRP contains various growth factors and cytokines that enhance the original ability of body tissue to repair and regenerate $(15,16)$. PRP therapy may be defined as self-preparation of blood plasma-enriched plasma.

The therapeutic benefit of PRP can be obtained not only from platelets but also from a combination of components and growth factors. Researchers have suggested that high levels of growth factors and cytokines in PRP will promote tissue rejuvenation and healing (13). Platelet densities of about 1 million platelets per microliter, which is about five times the normal level, have tissue-repair effects (16), but 
the PRP level in this study was only $344 \%$ greater than normal. The role of PRP was promised as a short-term agent to support simultaneous healing of several musculoskeletal tissues after trauma or selective surgery (16). In this study, the results show that it is possible to perform tracheal reconstruction in rabbits treated with PRP after tracheal transplantation.

\section{Acknowledgements}

This research was supported by Basic Science Research Program through the National Research Foundation of Korea (NRF) funded by the Ministry of Education (2015R1D1A1A01060583), Republic of Korea.

\section{References}

1 Delaere PR: Tracheal transplantation. Curr Opin Pulmon Med 18: 313-320, 2012.

2 Delaere P and Van Raemdonck D: Tracheal replacement. J Thorac Dis 8: S186-196, 2016.

3 Mercier O, Kolb F and Dartevelle PG: Autologous tracheal replacement surgical technique and outcomes. Thorac Surg Clin 28: 347-355, 2018.

4 Cho K, Kim JM, Kim MH, Kang SS, Kim G and Choi SH: Scintigraphic evaluation of osseointegrative response around calcium phosphate-coated titanium implants in tibia bone: Effect of platelet-rich plasma on bone healing in dogs. Eur Surg Res 51: 138-145, 2013.

5 Oh DS, Cheon YW, Jeon YR and Lew DH: Activated plateletrich plasma improves fat graft survival in nude mice: A pilot study. Dermatol Surg 37: 619-625, 2011.

6 Maia L and de Souza MV: Components rich in platelets used in wound healing tendon, ligaments and osteo-articular diseases of animals. Ciência Rural 39: 1267-1274, 2009.
7 Ghorbani A, Dezfouli AA, Shadmehr MB, Pejhan S, Saghebi SR, Ghare-Daghi A, Farzanegan $\mathrm{R}$ and Jahanshahi N: A Proposed grading system for post-intubation tracheal stenosis. Tanaffos 11: 10-14, 2012.

8 Auchincloss $\mathrm{HG}$ and Wright $\mathrm{CD}$ : Complications after tracheal resection and reconstruction: Prevention and treatment. J Thoracic Dis 8: S160-S167, 2016.

9 Zanin M, Baviskar P, Webster R and Webby R: The interaction between respiratory pathogens and mucus. Cell Host Microb 19(2): 159-160, 2016.

10 Delaere PR, Liu ZY, Hermans R, Sciot R and Feenstra L: Experimental tracheal allograft revascularization and transplantation. J Thorac Cardiovasc Surg 110: 728-737, 1995.

11 Coraux C, Hajj R, Lesimple P and Puchelle E: Repair and regeneration of the airway epithelium. Med Sci 21: 1063-1069, 2005.

12 Delaere PR, Liu Z, Sciot R and Welvaart W: The role of immunosuppression in the long-term survival of tracheal allografts. Arch Otolaryngol Head Neck Surg 122: 1201-1208, 1996.

13 Boswell SG, Cole BJ, Sundman EA, Karas V and Fortier LA: Platelet-rich plasma: a milieu of bioactive factors. Arthroscopy 28: 429-439, 2012.

14 Dhurat R and Sukesh M: Principles and methods of preparation of platelet-rich plasma: A review and author's perspective. J Cutan Aesthet Surg 7: 189-197, 2014.

15 Marwah M, Godse K, Patil S and Nadkarni N: Is there sufficient research data to use platelet-rich plasma in dermatology? Int $\mathrm{J}$ Trichol 6: 35-36, 2014.

16 Dhillon RS, Schwarz EM and Maloney MD: Platelet-rich plasma therapy - Future or trend? Arthritis Res Ther 14(4): 219, 2012.

Received August 16, 2018

Revised September 19, 2018

Accepted September 21, 2018 